\title{
THE CATEGORY OF COFINAL TYPES. I
}

\author{
BY \\ SEYMOUR GINSBURG AND J. R. ISBELL $\left(^{1}\right)$
}

Introduction. Briefly, this paper introduces a category $\mathscr{C}$ which we call the category of cofinal types. We construct a concrete representation $\mathscr{C}_{1}$ of $\mathscr{C}$, and we determine explicitly the part of $\mathscr{L}_{1}$ corresponding to the cofinal types of bases of open sets in regular topological spaces. These types have a privileged position in $\mathscr{C}$, and we call them canonical types. Succeeding papers in this series will develop further machinery, particularly for the cofinal types of directed sets.

The interest of the results depends heavily on the appropriateness of our definitions of convergent functions and equivalence of convergent functions between partially ordered sets (see below, before and after 1.1). In directed sets, a function $f: P \rightarrow Q$ is convergent if and only if it takes every cofinal subset to a cofinal subset; in general, $P$ and $Q$ may have branching structure which $f$ must respect. Equivalence of the convergent functions $f: P \rightarrow Q$ and $g: P \rightarrow Q$ means, except for some trivialities, that every function $h \subset f \cup g$ (i.e., every value $h(p)$ is $f(p)$ or $g(p)$ ) is convergent. The relation gives us a quotient $\mathscr{C}$ of the category $\mathscr{A}$ of all partially ordered sets and convergent functions. Two objects of $\mathscr{A}$ become isomorphic in $\mathscr{C}$ if and only if they are cofinally similar.

We adapt a construction $F$ from [2] to make several functors $F, F^{0}, F^{*}$ on $\mathscr{A}$ such that the quotient category is $\mathscr{C} . F^{*}$ induces a dual embedding of $\mathscr{C}$ in the category $\mathscr{B}^{*}$ of all complete Boolean algebras and complete homomorphisms, and a duality between $\mathscr{B}^{*}$ and the full subcategory of canonical types in $\mathscr{C}$. There is also a dual embedding of $\mathscr{B} \mathscr{B}^{*}$ in $\mathscr{A}$, giving a retraction $F: \mathscr{A} \rightarrow \mathscr{A}$ whose image is isomorphic with $\mathscr{C}$.

We are indebted to the referee for twenty-one constructive suggestions.

1. Convergence and equivalence. As we have remarked elsewhere, for convergence it is small sets that matter. It is convenient to define $y$ to be a successor of $x$ in a partially ordered set $P$ in case $y \leqq x$. (The reverse convention would require at least one reversal of order in 2.1-2.5 below.) As is customary, we call a set residual in $P$ if it contains all successors of its elements, cofinal if it meets every nonempty residual subset of $P$.

A subset of a partially ordered set will be called total if it contains a cofinal residual set. A covering $\left\{V_{\alpha}\right\}$ of a partially ordered set is called normal if its

Received by the editors March 23, 1964.

( ${ }^{1}$ ) Second named author supported by Office of Naval Research and National Science Foundation. 
trace $\left({ }^{2}\right)$ on some total subset has a refinement consisting of residual sets. In other words, the union of the residual sets $R$ such that for some $\alpha, R \subset V_{\alpha}$, is cofinal. A function between partially ordered sets is convergent if the inverse image of every total set is total and the inverse image of every normal covering is normal.

1.1. If $f: P \rightarrow Q$ is convergent, then for every residual subset $R$ of $Q$ there is a total subset $T$ of $P$ such that $f^{-1}(R) \cap T$ is residual.

Proof. Cover $Q$ with $R$ and $Q-R$. This is normal; for the union $U$ of $R$ and all residual sets disjoin from $R$ is residual and cofinal, and the trace of $\{R, Q-R\}$ on $U$ consists of residual sets. Hence for some total $T$ in $P$, $\left\{f^{-1}(R) \cap T, f^{-1}(Q-R) \cap T\right\}$, a partition of $T$, has a refinement consisting of residual sets. So $f^{-1}(R) \cap T$ is residual.

REMARKs. (1) The definition of convergence is analogous (and related [3]) to one definition of uniform continuity. The analogy could be sharpened by using normal "coverings" which cover only a total set; if the inverse images of these are coverings of the same sort, then the inverse images of total sets must be total.

(2) The conclusion of 1.1 is equivalent to the condition that inverse images of finite normal coverings are normal. This and the condition that inverse images of total sets are total would suffice for much of what follows; but these conditions can be satisfied by a function from a directed set to an everywhere branching set, though no such function is convergent. (A partially ordered set $Q$ is everywhere branching [1] if every element $q$ has two successors $r_{1}, r_{2}$ which have no common successor. Then if $P$ is a directed set and $f: P \rightarrow Q$ a function, there is a nonempty residual set $R$ of successors of $q$ (viz. the set of successors of one $r_{j}$ ) such that $f^{-1}(R)$ contains no nonempty residual subset of $P$. All of these residual sets and all singletons contained in $Q$ make a normal covering; hence $f$ is not convergent. On the other hand, if $P$ is an ultrafilter of subsets of $Q$ containing the filter of total subsets, and $g$ a choice function from $P$ to $Q$, one can check that the inverse images of total sets are total and the inverse images of finite normal coverings are normal.)

Two convergent functions $f: P \rightarrow Q, g: P \rightarrow Q$ are equivalent if for each residual subset $R$ of $Q$ there is a total subset $T$ of $P$ such that $f^{-1}(R) \cap T$ $=g^{-1}(R) \cap T$.

(3) This relation implies (as was mentioned in the introduction) that every "mixture" $h \subset f \cup g$ is convergent, and the converse is true for sets without minimal elements. (If $f, g$ are equivalent and $h \subset f \cup g$ then $h^{-1}(U)$ contains the total set $f^{-1}(U) \cap g^{-1}(U)$, for each total $U$ in $Q$. For any normal covering $\left\{S_{\alpha}\right\}$ of $Q$ there are residual sets $R_{\alpha} \subset S_{\alpha}$ whose union $R$ is cofinal, and

$\left({ }^{2}\right)$ When $\left\{U_{\alpha}\right\}$ is a covering of $P$ and $S$ is a subset of $P$, the trace of $\left\{U_{\alpha}\right\}$ on $S$ is the covering $\left\{U_{\alpha} \cap S\right\}$. 
total sets $T_{\alpha}$ in $P$ such that $f^{-1}\left(R_{\alpha}\right) \cap T_{\alpha}=g^{-1}\left(R_{\alpha}\right) \cap T_{\alpha}$. The sets $R_{\alpha}$, with $Q-R$, form a normal covering of $Q$. Hence every element $p$ of $P$ has a successor $v$ in a residual set $V$ contained in some $f^{-1}\left(R_{\alpha}\right) ; v$ has a successor $w$ whose successors form a residual set $W \subset V \cap T_{\alpha}$, so that $h(W) \subset R_{\alpha}$. That is, the residual subsets of sets $h^{-1}\left(R_{\alpha}\right)$ have cofinal union, and $h$ is convergent. Conversely, suppose $P$ has no minimal elements; for nonequivalent $f, g$, there is some $f^{-1}\left(R_{0}\right)-g^{-1}\left(R_{0}\right)$, or $g^{-1}\left(R_{0}\right)-f^{-1}\left(R_{0}\right)$, whose complement is not total; one uses Zorn's Lemma to split this set into two parts $A, B$, having the same property, so that the function which is $f$ on $A$ and $g$ on $P-A$ is certainly nonconvergent.)

(4) If $f: P \rightarrow Q$ is convergent and $g: P \rightarrow Q$ satisfies $g(x) \leqq f(x)$ for all $x \in P$, then $g$ is convergent and equivalent to $f$.

(5) Partially ordered sets and convergent functions form a category; that is, identity functions $1: P \rightarrow P$ are convergent, and compositions of convergent functions are convergent. The isomorphisms of this category (see [4], for example, for basic notions of category and functor theory) are a curious class of functions. We are more interested in classification up to equivalence; and we note that if $f: P \rightarrow Q$ and $g: P \rightarrow Q$ are equivalent convergent functions then for any convergent $h: Q \rightarrow R$ and $e: M \rightarrow P$, hfe is equivalent to hge.

We define a type equivalence of two partially ordered sets $P, Q$, as a convergent mapping $f: P \rightarrow Q$ such that there exists a convergent mapping $g: Q \rightarrow P$ such that $f g$ and $g f$ are equivalent to identity mappings.

1.2. Theorem. There exists a type equivalence $f: P \rightarrow Q$ if and only if $P$ and $Q$ are cofinally similar $\left({ }^{3}\right)$.

Proof. If $P$ and $Q$ are embeddable as cofinal subsets of a set $R$, then one has convergent functions $f: P \rightarrow Q$ and $g: Q \rightarrow P$ such that $g f(x) \leqq x$ and $f g(y) \leqq y$ for all $x$ in $P, y$ in $Q$; so $f$ is a type equivalence (Remark (4)). Conversely, if $f: P \rightarrow Q$ and $g: Q \rightarrow P$ are convergent and their compositions are equivalent to identities, define a quasi-ordering of the disjointed union of $P$ and $Q$ as follows. Define $x \leqq x^{\prime}$ in $P$ and $y \leqq y^{\prime}$ in $Q$ by the given orderings. Put $y \leqq x$ in case $y^{\prime} \leqq y$ implies $g\left(y^{\prime}\right) \leqq x$, but for at least one $x^{\prime} \leqq x$, $f\left(x^{\prime}\right) \geqq y$. Similarly $x \leqq y$ if $f$ takes all successors of $x$ to successors of $y$ but $g$ takes some successor $y^{\prime}$ of $y$ to $g\left(y^{\prime}\right) \geqq x$. This relation is reflexive since $P$ and $Q$ are partially ordered. For transitivity, supposing $x^{\prime} \leqq y \leqq x$, there is $y^{\prime} \leqq y$ such that $g\left(y^{\prime}\right) \geqq x^{\prime}$, and necessarily $g\left(y^{\prime}\right) \leqq x$; since $P$ is partially ordered, $x^{\prime} \leqq x$. Then trivial arguments for other cases establish that $P \cup Q$ is quasi-ordered. Identifying any pairs where $x \leqq y \leqq x$, we get a partially

$\left({ }^{3}\right)$ Two partially ordered sets are defined to be cofinally similar if there exists a third partially ordered set in which both of them can be embedded as cofinal subsets. It is known that this is an equivalence relation, and Theorem 1.2 is known for directed sets [5, p. 13]. 
ordered set $R$ containing $P$ and $Q$. (No further identifications are required, for $x \leqq y \leqq x$ implies $y=f(x)$ and $x=g(y)$.) To see that $P$ is cofinal, consider any $y$ in $Q$ and the set $A$ of successors of $y$ in $Q$. By $1.1, f^{-1}(A)$ consists of a residual set $B$ and a negligible set $f^{-1}(A)-T$, where $T$ is total. Then $g^{-1}(T)$ is total, and $g^{-1} f^{-1}(A)$ coincides on some total set with $A$. These two sets intersect in $g^{-1}(B)$, which thus contains a nonempty residual subset $S$ of $A$. Let $y^{\prime} \in S$. As $g\left(y^{\prime}\right) \in B$ and $f(B) \subset A, g\left(y^{\prime}\right) \leqq y$. By a parallel argument, $Q$ is also cofinal in $R$, and the proof is complete.

2. The functor $F$. A maximal residual set is defined [2] as a residual set that is not a cofinal proper subset of any residual set. The maximal residual subsets of an everywhere branching set, ordered by inclusion, form a complete atomless Boolean algebra [2]. We need

2.1. The maximal residual subsets of any partially ordered set form a complete Boolean algebra.

(Clarification: If one admits the empty partially ordered set, one must admit the corresponding degenerate Boolean algebra.)

Proof. A trivial check shows that every intersection of maximal residual subsets is maximal residual; so one has a complete lattice. It is complemented, the complement of $A$ being the union of all residual sets disjoint from $A$. Finally, relative complements are unique; if $A \cap B=C$ and $A \vee B=D$, we show that $B$ is exactly the union of all residual sets $E \subset D$ such that $A \cap E \subset C$. Since $B$ is itself such a set, it is contained in their union $U$. As $U$ is residual and $B$ is maximal residual, it will suffice to show that $B$ is cofinal in $U$. Suppose it is not. Then some nonempty residual set $E \subset U$ is disjoint from $B$. As $A \cap E \subset C \subset B$, this implies that the union $E^{\prime}$ of all residual sets disjoint from $E$ contains $A$ as well as $B$. But $E^{\prime}$ is maximal residual, for a residual set in which $E^{\prime}$ is cofinal must still be disjoint from $E$. As $E^{\prime}$ does not contain $D$, this contradicts a hypothesis, and completes the proof.

The utility of maximal residual sets for representing equivalence classes of mappings can be brought into view by introducing an equivalence relation on all subsets. We call subsets $R, S$ of a partially ordered set $P$ equivalent if for some total subset $T$, every element of $R \cap T$ has a successor in $S \cap T$ and every element of $S \cap T$ has a successor in $R \cap T$. A definition of order for equivalence classes suggests itself, but need not be analyzed, for each equivalence class contains exactly one maximal residual set. For the proof: there is at most one, because given two different maximal residual sets, one contains a nonempty residual set disjoint from the other, so they are not equivalent. On the other hand, for any set $S$, let $\phi S$ be the union of all residual sets $R$ in which $S \cap R$ is cofinal. Clearly $\phi S$ is maximal residual. Consider also the union $Z$ of all residual sets disjoint from $S$. Then $Z \cup \phi S$ is residual. It is also cofinal, for if $x$ has no successor in $Z, x$ has no successor 
which has no successor in $S$, and $x \in \phi_{\phi} S$. Then $Z \cup \phi S$ is a total set in which every element of $S$ or $\phi S$ has a successor in the other; $S$ is equivalent to $\phi S$.

The operation $\phi$ is used through the rest of the paper.

2.2. Convergent mappings $f, g$ on $P$ to $Q$ are equivalent if and only if for every (maximal) residual subset $R$ of $Q, \phi f^{-1}(R)=\phi g^{-1}(R)$.

Proof. With the parenthesis omitted, the equivalence is immediate. But the maximal residual sets suffice, because every residual set $R$ differs from $\phi R$ only in the complement of a total set.

We can now describe the following diagram of categories and functors:

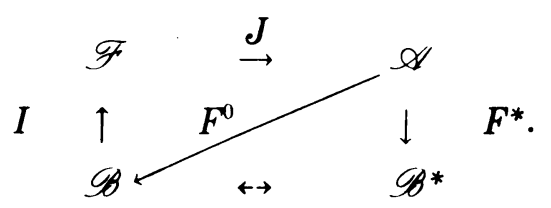

Here $\mathscr{A}$ is the category of partially ordered sets and convergent mappings. $\mathscr{B B}^{*}$ is the category of complete Boolean algebras and complete (unit-preserving) homomorphisms. $F^{*}(P)$ is the algebra of maximal residual subsets of $P$, and $F^{*}(f)$ is the function $\phi f^{-1}$ of 2.2 .

2.3. $F^{*}$ is a contravariant functor with values in $\mathscr{B}^{*}$.

Proof. $F^{*}$ is functorial because for $f: P_{1} \rightarrow P_{2}$ and $g: P_{2} \rightarrow P_{3}, f^{-1}$ takes equivalent residual sets to equivalent sets and $\phi f^{-1} \phi g^{-1}=\phi f^{-1} g^{-1}$ on $F^{*}\left(P_{3}\right)$. The values $\phi f^{-1}$ are complete homomorphisms because they take partitions to partitions. (A partition of a Boolean algebra is a set of its elements $x_{\alpha}$ whose least upper bound is 1 but with $x_{\alpha} \wedge x_{\beta}=0$ for $\alpha \neq \beta$.)

We leave the diagram to describe one representation of the category dual to $\mathscr{B} \mathscr{B}^{*}$. ( $\mathscr{B}$ is a more convenient representation of it $\left({ }^{4}\right)$.) A canonical mapping of a complete Boolean algebra $A$ to a complete Boolean algebra $B$ is a function $k: A \rightarrow B$, not necessarily a Boolean homomorphism, such that (1) $k$ is completely join-preserving, (2) $x \neq 0$ in $A$ implies $k(x) \neq 0$, and (3) for every ideal $I$ of $A, k(I)$ is an ideal.

Suppose $h: B \rightarrow A$ is a complete homomorphism. We define $\delta(h)=k$ : $A \rightarrow B$ by $k(x)=\bigwedge[y: h(y) \geqq x]$. Inversely, if $k: A \rightarrow B$ is a canonical mapping, we define $\delta^{-}(k)=h: B \rightarrow A$ by $h(y)=\bigvee[x: k(x) \leqq y]$. Let $\delta(A)$ $=\delta^{-}(A)=A$.

2.4. $\delta$ and $\delta^{-}$are mutually inverse contravariant functors, establishing a duality between the category of complete Boolean algebras and complete homomorphisms and the category of complete Boolean algebras and canonical mappings.

( 4 ) Stone duality provides a representation by extremally disconnected compact spaces and continuous open mappings. Our alternative representations can be derived from that one by the observations (a) the image of an open-closed set is open-closed; (b) the image of a nonempty set is nonempty. 
We shall omit the routine computational proof.

If $A$ is a complete Boolean algebra, let $A^{0}$ be the partially ordered set $A-\{0\}$; if $P$ is a partially ordered set, let $F^{0}(P)$ be $F^{*}(P)^{0}$, the family of nonempty maximal residual subsets of $P$. Call these partially ordered sets $A^{0}$ canonical sets. Define a canonical mapping $j: A^{0} \rightarrow B^{0}$ as the restriction to $A^{0}$ of a canonical mapping $k: A \rightarrow B$. Evidently

2.5. The category $\mathscr{B}$ of canonical sets and canonical mappings is dual to the category $\mathscr{B B}^{*}$.

Obviously, also,

2.6. Every canonical mapping is convergent.

The referee pointed out that the following lemma, which we originally included in the proof of 2.8, applies to 2.6 and simplifies checking it. A function is called isotone if it preserves the relation $\leqq$, or equivalently, the inverse images of residual sets are residual. An isotone function is convergent if (and only if), under it, the inverse images of total sets are total. We omit the simple proof.

Now the diagram is quickly filled in. $\mathscr{F}$ is the category of canonical sets and convergent mappings. $I: \mathscr{B} \rightarrow \mathscr{F}$ and $J: \mathscr{F} \rightarrow \mathscr{A}$ are identical embeddings. $F^{0}: \mathscr{A} \rightarrow \mathscr{B}$ is defined from $\delta F^{*}$ by removing 0 from the algebras.

By 2.2 and 2.4, $F^{0}$ identifies equivalent mappings but not nonequivalent mappings. Thus the quotient category $\mathscr{C}$ of $\mathscr{A}$ by $F^{0}$ is exactly the category of partially ordered sets and equivalence classes of convergent mappings. We call this the category of cofinal types. The full subcategory which is the image of $\mathscr{F}$ (the quotient of $\mathscr{F}$ by $F^{0} J$ ) is the category of canonical cofinal types. We have:

2.7. Theorem. The category of cofinal types is embeddable in the category of sets.

Let $F=J I F^{0}: \mathscr{A} \rightarrow \mathscr{\&}$.

2.8. Theorem. For every partially ordered set $P$ there exists a convergent mapping $\psi_{P}: P \rightarrow F(P)$ such that

(i) $\psi_{P}$ is an order isomorphism if $P$ is a canonical set;

(ii) $\psi_{Q} f$ is equivalent to $F(f) \psi_{P}$ for every $f: P \rightarrow Q$ in $\mathscr{A}$;

(iii) $\psi_{Q} f=F(f) \psi_{P}$ if $f$ is canonical.

Proof. For each $p$ in $P$ let $A(p)$ be the set of all successors of $p$ (including $p$ itself), and $\psi_{P}(p)=\phi A(p)$. Since $A(p)$ is a nonempty residual set, so is $\psi_{P}(p)$; i.e., the values are in $F(P)$. Note that $\psi_{P}$ is isotone. If $T$ is total in $F(P)$, then for every $p$ in $P$ the set $\phi A(p)$ contains some $t \in T$. As $A(p)$ is cofinal in $\phi A(p)$, there is an element $p^{\prime}$ of $A(p) \cap t$. Then $A\left(p^{\prime}\right) \subset t$, $\psi_{P}\left(p^{\prime}\right) \leqq t$; thus $\psi_{P}^{-1}(T)$ is total. Therefore $\psi_{P}$ is convergent.

If $P$ is a canonical set, we can establish that $\psi_{P}$ is one-to-one by showing 
that each set $A(p)$ is maximal residual. Now each residual set $B$ properly containing $A(p)$ has an element $p^{*}$ whose infimum with the Boolean complement $p^{\prime}$ is not 0 , and $p^{*} \wedge p^{\prime}$ is a member of $B$ having no successor in $A(p)$. Next we prove $\psi_{P}$ is onto. First, each nonempty residual set $C$ is cofinal in $A(p)$, where $p$ is the Boolean supremum of $C$. To prove this, suppose $x$ has no successor in $C$. Then $x \neq 1$, so we have a complement $x^{\prime}$ in $P$. Every member $c$ of $C$ must be in $A\left(x^{\prime}\right)$, as otherwise $c \wedge x$ would be a successor of $x$ in $C$. Hence $x^{\prime} \geqq p$, and $x$ is not in $A(p)$; in $A(p), C$ is cofinal. For maximal residual $C$ this means $C=A(p)=\psi_{P}(p)$.

To verify equivalence of $\psi_{Q} f$ and $F(f) \psi_{P}$, one can apply 2.2 , which requires $\phi f^{-1} \psi_{Q}^{-1}=\phi \psi_{P}^{-1} F(f)^{-1}$. Each element of the domain $F(F(Q))$ has the form $A(S), S \in F(Q)$; one finds that $f^{-1} \psi_{Q}^{-1}$ takes it to $f^{-1}(S)$ and $\psi_{P}^{-1} F(f)^{-1}$ takes it to $\phi f^{-1}(S)$.

For canonical mappings $f: P \rightarrow Q$, equality of $\psi_{Q} f$ and $F(f) \psi_{P}$ follows from their equivalence in view of previous results, especially 2.4 .

A number of corollaries can be drawn concerning the various categories; for example, $F F: \mathscr{A} \rightarrow \triangle$ is naturally equivalent to $F$. We number two of them.

2.9. Corollary. The functor $F^{*} J$ induces a duality between the category of all canonical cofinal types and the category of all complete Boolean algebras and complete homomorphisms.

2.10. Corollary. If $P$ has the cofinal type of a canonical set then $\psi_{P}$ : $P \rightarrow F(P)$ is a type equivalence.

2.10 follows from the equivalence of $\psi_{Q} f$ and $F(f) \psi_{P}$, where $f$ is a type equivalence to a canonical set $Q$.

2.11. A sufficient condition for $P$ to be cofinally similar to a canonical set is that every nonempty residual subset of $P$ contains a nonempty maximal residual set. This condition is satisfied by the family of all nonempty open subsets of a regular topological space, or by any cofinal subset of it.

Proof. For any $P$, the nonempty residual sets form a partially ordered set in which the sets $A(p)$ form a cofinal subset isomorphic with $P$. The given condition means that $F(P)$ is also cofinal. Finally, in any topological space, the maximal residual sets of nonempty open sets are precisely the sets $A(S)$ where $S$ is the interior of a closed set; if the space is regular, they are cofinal in the family of all nonempty residual sets.

REMARK. Trivial examples show that the sufficient condition of 2.11 is not necessary. However, the canonical sets themselves all satisfy it, and indeed they are all isomorphic, by Stone duality, with bases of open sets in suitable regular spaces.

3. Connecting remarks. We noted that 1.2 is known for directed sets. 
Tukey's definitions are different; for the connection, one needs part of the following result.

3.1. A function from a partially ordered set $P$ to a directed set $Q$ is convergent if and only if the image of every subset of the domain which is cofinal in some nonempty residual set is cofinal in the range. Any two convergent functions on $P$ to $Q$ are equivalent.

We omit the routine proof. The referee caught our careless misstatement that the images of (only) cofinal sets must be cofinal; it seems worth mentioning, because that simpler criterion is valid if $P$ as well as $Q$ is directed. Therefore, the structure we have defined on the class of all cofinal types of directed sets (a full subcategory of $\mathscr{C}$ ) is essentially the same as the structure defined by Tukey [5] (a partially ordered class).

Thus we have an extension of Tukey's structure over nondirected sets. Several such extensions have previously been defined by Day [1], all featuring relations between partially ordered sets rather than mappings establishing the relations. The present approach differs from all of Day's in stressing the mappings. However, the main difference is more fundamental; we regard the branching structure as essential, while in [1] a partially ordered set seems to be primarily a carrier for functions into topological spaces converging to a point. Our convergent functions [3] may converge all over a space. The value of this notion of convergence is proved, so far, only by one application; but it seems to the point to mention Day's result that in his sense, directed sets are sufficient for convergence [1]. No such reduction is possible for the convergence of [3].

\section{REFERENCES}

1. M. Day, Oriented systems, Duke Math. J. 11 (1944), 201-229.

2. S. Ginsburg, A class of everywhere branching sets, Duke Math. J. 20 (1953), 521-526.

3. J. Isbell, Supercomplete spaces, Pacific J. Math. 12 (1962), 287-290.

4. S. MacLane, Homology, Academic Press, New York, 1963.

5. J. Tukey, Convergence and uniformity in topology, Annals of Mathematics Studies No. 2, Princeton Univ. Press, Princeton, N. J., 1940.

System Development Corporation, Santa Monica, California

INSTITUTE FOR ADVANCED STUDY, Princeton, New Jersey 